\title{
All-optical, transform-limited and high mark-space-ratio soliton pulse train generation using both CDPF ad NOLM
}

Yujun, Qian; Povlsen, Jørn Hedegård; Varming, Poul

Published in:

CLEO'99

Link to article, DOI:

10.1109/CLEO.1999.834218

Publication date:

1999

Document Version

Publisher's PDF, also known as Version of record

Link back to DTU Orbit

Citation (APA):

Yujun, Q., Povisen, J. H., \& Varming, P. (1999). All-optical, transform-limited and high mark-space-ratio soliton pulse train generation using both CDPF ad NOLM. In CLEO'99 (pp. 299-300). IEEE.

https://doi.org/10.1109/CLEO.1999.834218

\section{General rights}

Copyright and moral rights for the publications made accessible in the public portal are retained by the authors and/or other copyright owners and it is a condition of accessing publications that users recognise and abide by the legal requirements associated with these rights.

- Users may download and print one copy of any publication from the public portal for the purpose of private study or research.

- You may not further distribute the material or use it for any profit-making activity or commercial gain

- You may freely distribute the URL identifying the publication in the public portal 


\section{CWF73}

\section{Analytic solutions for broadband pulse generation from near-infrared to near- ultraviolet by induced-phase modulation in a gas-filled hollow waveguide}

Naoki Karasawa, Ryuji Morita, Mikio Yamashita, Department of Applied Physics, Hokkaido University, Kita-13, Nishi-8, Kita-Ku, Sapporo, 060-8628 Japan, and CREST, Japan Science and Technology Corporation (JST); E-mail:

karasawa@eng.hokudai.ac.jp

Generation of an ultra-broadband optical pulse with a fluent frequency-dependency of the phase is important, for example, for shaping multi-wavelength optical pulses and monocyclization. When two optical pulses, one fundamental, the other generated by second harmonic generation, are co-propagated in a nonlinear waveguide, induced-phase modulation (IPM) between them, as well as self-phase modulation (SPM), can be used to broaden the spectrum of each pulse. ${ }^{1}$ Since the second-harmonic wave as well as the fundamental wave are generated from one common femtosecond pulse, the carrier-phase differ-
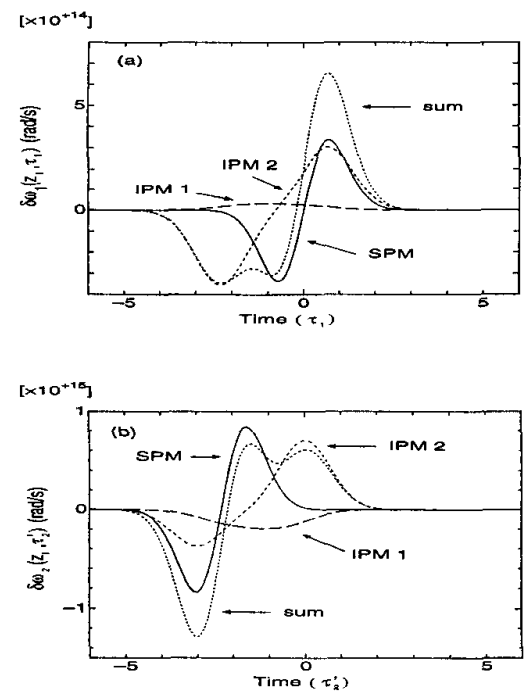

CWF73 Fig. 1. Calculated chirps versus normalized time for (a) pulse 1 and (b) pulse 2 when dispersion terms are neglected. Two input pulses are given as $A_{1}(0, T)=\sqrt{P_{1}} \exp \left[-T^{2} /\left(2 T_{01}^{2}\right)\right]$ and $\left.A_{2}\left(0, T^{\prime \prime}\right)=\sqrt{P_{2}} \exp \left[-T^{\prime}-T_{d 2}\right)^{2} /\left(2 T_{02}^{2}\right)\right]$ where $P_{i}$ is a peak power, $T_{0 i}$ is a pulse width, and $T_{d 2}$ is a delay time of the pulse 2 with respect to the pulse 1 and $\tau_{i}=T / T_{0 i}, \tau_{i}^{\prime}=T^{\prime} / T_{0 i}$ where $T=t-$ $\beta_{11} z, T^{\prime}=t-\beta_{12} z$ and $\beta_{1 i}$ is the reciprocal of a group velocity of the pulse $i$. Solid lines show chirps from SPM, long and short dashed lines show chirps from IPM, and dotted lines show the total chirps. Pulse centers are at 0 for (a) and -2.32 for (b). In calculations, $\omega_{1}$ is the fundamental wave and $\omega_{2}$ is the second-harmonic wave of a Ti:sapphire laser system $\left(\lambda_{1}=790 \mathrm{~nm}\right)$. These are propagated in an argon-filled $(300 \mathrm{~K}, 3.3 \mathrm{~atm})$ capillary fiber with a radius of $50 \mu \mathrm{m}$ and a length of $29.2 \mathrm{~cm}$. Also, $P_{1}=P_{2}=1.29 \mathrm{GW}, T_{01}=T_{02}=$ $30 /(2 \sqrt{\ln 2}) f_{s}\left(\tau_{1}=\tau_{2}, \tau_{1}^{\prime}=\tau_{2}^{\prime}\right), n_{2}=3.234 \times$ $10^{-23} \mathrm{~m}^{2} / \mathrm{W}$ and $T_{d 2}=-41.8 \mathrm{fs}\left(T_{d 2} \simeq z_{l} d\right)$ are used. ence between their waves is constant and these can be synthesized constructively after propagation.

In this research, we analyzed the case where a capillary fiber filled with noble gas ${ }^{2}$ is used as an almost-dispersionless, nonlinear waveguide. Since the optical field is not completely confined in the glass capillary during propagation, the loss always occurs in this method. We consider a case where two optical pulses with different center frequencies and widths are co-propagating in a capillary fiber. The analytic equations for nonlinear chirps with loss, but without dispersion are obtained for two Gaussian input-pulses using slowly varying envelope approximations ${ }^{3}$ and those are shown in Fig. 1.

The optimum delay between two pulses and powers required for covering spectrum between two pulses can be estimated from analytical solutions obtained in this study. For the case of co-propagating fundamental $\left(\lambda_{1}=\right.$ $790 \mathrm{~nm}$, pulse width $30 \mathrm{fs}$ ) and second harmonic $\left(\lambda_{2}=395 \mathrm{~nm}\right.$, pulse width $\left.30 \mathrm{fs}\right)$ waves from a Ti:sapphire laser system with a capillary fiber filled with argon at 3.3 bar and $300 \mathrm{~K}$, the optimum delay time is obtained to be $-41.8 \mathrm{fs}$ for which both pulses meet near the fiber exit end. The required power is calculated to be $P_{1}$ $=P_{2}=1.29 \mathrm{GW}$ assuming $P_{1}=P_{2}$.

To verify the above results with the dispersion terms, numerical calculations including second- and third-order dispersion terms are also performed and the result is shown in Fig. 2. This figure shows that the spectra of fundamental and second-harmonic waves are just overlapping as expected. From this spectrum $(333 \sim 1000 \mathrm{~nm})$, a compressed pulse duration using a 256-channel spatial phase modulator according to Ref. 1 is estimated to be $3.06 \mathrm{fs}$ and it is shown by a dotted line in Fig. 2.

In conclusion, ultra-broadband optical pulse generation utilizing SPM and IPM in a gas-filled hollow waveguide has been analyzed and the results have been verified by numerical calculations including dispersion effects. As an example, for the experimentally realizable pa-

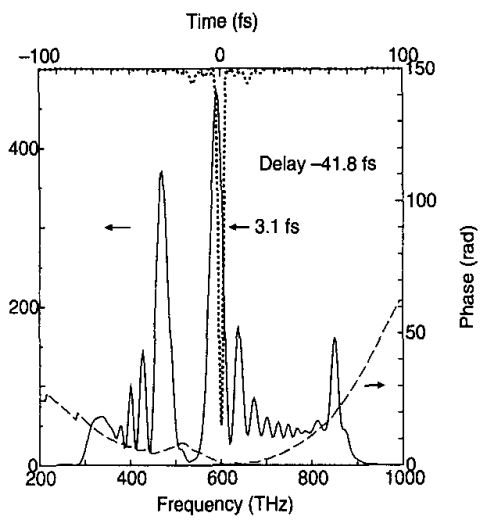

CWF73 Fig. 2. Calculated intensity (solid line) and phase (dashed line) versus frequency of the fundamental and second-harmonic waves after propagating an argon-filled capillary fiber under the condition $P_{1}=P_{2}=1.29 \mathrm{GW}, T_{d 2}=$ $-41.8 \mathrm{fs}$. Calculated intensity versus time of the compressed pulse after its phase is compensated for by the spatial phase modulator is shown in the reverse direction (dotted line). rameters utilizing the fundamental and the second harmonic waves from a Ti:sapphire laser system with an Ar-filled capillary fiber, the optimum delay time and the required pulse peak powers have been obtained.

Those calculations show that it is possible to generate the ultra-broadband quasi-linear chirped optical pulse that covers fully the spectrum between fundamental and second harmonic waves of Ti:sapphire laser systems by this method. An experimental verification of this theory is under way in our laboratory.

1. (a) M. Yamashita, H. Sone, and R. Morita, Jpn. J. Appl. Phys. 35, L1194 (1996), (b) M. Yamashita, H. Sone, R. Morita, and H. Shigekawa, to be published in IEEE ). Quantum Electron.

2. M. Nisoli, S. De Silvestri, and O. Svelto, Appl. Phys. Lett. 68, 2793 (1996).

3. G.P. Agrawal, Nonlinear Fiber Optics (Academic Press, San Diego, 1989).

\section{CWF74}

All-optical, transform-limited and high mark-space-ratio soliton pulse train generatlon using both CDPF and NOLM

Y. Qian, J.H. Povlsen, P. Varming, Center for Communications, Optics and Materials

(COM), Technical University of Denmark, Building 349, DK-2800, Denmark; E-mail: qy@com.dtu.dk

Transform-limited pulse train at multiples of $10 \mathrm{GHz}$ repetition frequency with high markspace-ratio (MSR: ratio of pulse FWHM to time cycle) has increasing applications in optical communications. Comblike dispersionprofiled fibre $(\mathrm{CDPF})^{1}$ is a promising compression scheme due to its simplicity and flexibility splicing alternatively the dispersion shifted fibres (DSF, dispersion as 0 at $1550 \mathrm{~nm}$ ) and the standard telecommunication fibres (STF, dispersion as $-20 \mathrm{ps}^{2} / \mathrm{km}$ ). But with CDPF or conventional NOLM alone, it's difficult to convert optical heat signal into transform-limited soliton train with MSR higher than 1:10. We propose here for the first time, to our knowledge, an effective all-optical transform-limited pulse generation configuration with both CDPF and NOLM, with output pulse MSR higher than 1:40 at multiples of $10 \mathrm{GHz}$.

The schematic system, shown in Fig. 1, consists of a DFB fibre laser beat source with amplifier, a CDPF, and a NOLM followed by chirp-compensation STF. Fibre nonlinearity, absorption and splicing losses are chosen as $3 \mathrm{~W}^{-1} \mathrm{~km}^{-1}, 0.25 \mathrm{~dB} / \mathrm{km}$ and $0.1 \mathrm{~dB} /$ splice, respectively. The split-step Fourier method is used and simulations are initially confirmed by the reported experimental results with different parameters. ${ }^{1-3}$ The SPM phase-shifts for the first and other DSFs segments are chosen as $0.40 \pi$ and $0.20 \pi$, respectively. 5 segments each for DSF and STF is used in CDPF. The average power of amplified beat signal is $100 \mathrm{~mW}$. The beat repetition rate can be changed by tuning 4 the DFB laser's dual-wavelength difference, i.e. $10 \mathrm{GHz} / 0.08 \mathrm{~nm}$. Figure 2 shows the frequency dependency of pulse evolution. With frequency higher than $40 \mathrm{GHz}$, all parameters 


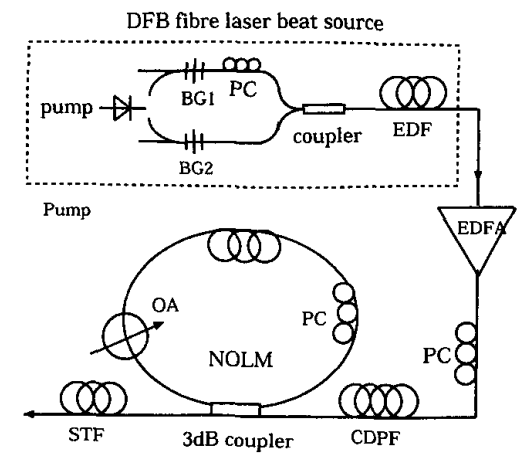

CWF74 Fig. 1. Schematic diagram of shortpulse generation system. OA, optical attenuator: PC, polarisation controller; $\mathrm{BG}$, Bragg grating; EDF, Er-doped fibre; EDFA, Er-doped fibre amplifier.

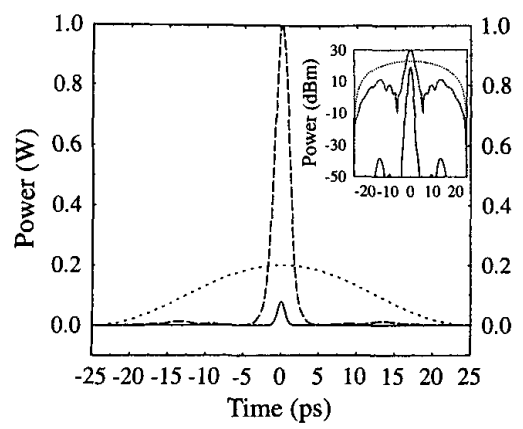

CWF74 Fig. 2. Pulse (before NOLM) evolution characteristics vs beat signal repetition rates. $R_{r m s}$ and $R_{f w h m}$ are RMS and FWHM compression ratios, respectively: $R_{\text {peak }}$ is the ratio of output to input peak power and $\delta_{\text {rms }}$ the RMS timebandwidth-product ratio relative to fundamental soliton.

change little and transform-limited soliton pulse train can be obtained with moderate MSR of about 1:8. Most of the reported experiments were performed in such cases. With frequency lower than $30 \mathrm{GHz}$, it's easy to obtain highly-compressed pulse train, but the pulse quality is not good. By incorporating NOLM's

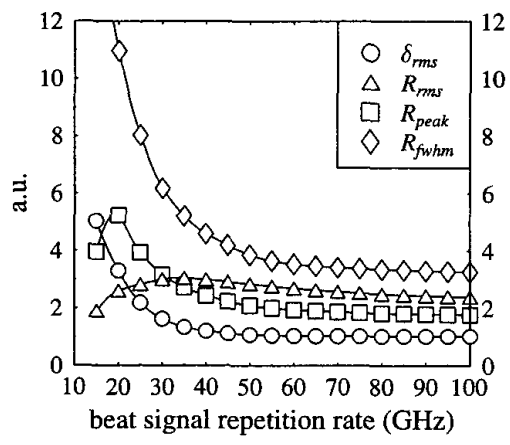

CWF74 Fig. 3. Pulse shapes of amplified beat signal (dot line), after CDPF (dashed line) and after NOLM (solid line) at $20 \mathrm{GHz}$. Inset is on $\log$ scale. efficient reshaping, highly-compressed, highquality pulse train could be obtained.

A NOLM with $1 \mathrm{~km}$ DSF and attenuation factor of 0.35 is chosen, followed by $11 \mathrm{~m}$ final STF. The pulse shapes are illustrated in Fig. 3 with inset on the log scale, at $20 \mathrm{GHz}$. After CDPF, the beat signal is highly-compressed with pulse MSR as 1:22. But the pulse's RMS product ratio $\delta_{\mathrm{rms}}$ is also as high as 3.2 (for fundamental soliton, $\delta_{\mathrm{rms}}=1$ ), implying poor quality with side-lobes in the inset. After propagating through NOLM and final STF, the pulse is further compressed with MSR as high as 1:45 and in addition, the side-lobes are $50 \mathrm{~dB}$ lower, with $\delta_{\text {rms }}$ of only 0.97 , indicating transform-limited pulse train formation.

In conclusion, a combined all-optical implementation of CDPF and NOLM is proposed for transform-limited solitonlike pulse generation with pulse MSR higher than 1:40, at multiples of $10 \mathrm{GHz}$. Relative experiment will be conducted and applied in optical communications.

1. S.V. Chernikov, J.R. Taylor, and R. Kashyap, "Comblike dispersion-profiled fibre for soliton pulse train generation," Opt. Lett. 19, 539-541 (1994).

2. S.V. Chernikov, J.R. Taylor, and R. Kashyap, "Integrated all-optical fiber source of a multigigahertz soliton pulse train," OFC'94 Technical Digest, 1994, paper TuG4.

3. D.K. Serkland, G.D. Gartoline, W.L. Kath P. Kumar, and A.V. Sahakian, "Rate multiplication of a $59-\mathrm{GHz}$ soliton source at $1550 \mathrm{~nm}$," J. Lightwave Technol., 16, 670677 (1998).

4. J. Hübner, P. Varming, and M. Kristensen, "Five wavelength DFB laser source for WDM systems," Electron. Lett. 28, 139 140 (1997).

\section{CWF75}

All-optical demultiplexing scheme based on an optical parametric loop mirror

Min-Yong Jeon, Hak Kyu Lee, Joon Tae Ahn, Dong Sung Lim, Do Il Chang,

Kyong Hon Kim, Electronics and

Telecommunications Research Institute,

Yusong, P.O. Box 106, Taejon 305-600 South Korea; E-mail: myjeon@etri.re.kr

In recent years, several application schemes based on four wave mixing (FWM) effect in optical parametric loop mirror (PALM), such as wavelength interchange, wavelength conversion and spectral inversion, have been proposed. ${ }^{1,2}$ The generated FWM wave appears to be relatively strong compared to the input signal and control beams used in those scheme, and can be easily separated out from the other beams. In this paper, we demonstrate, the first time to our knowledge, an all optical demultiplexing of $10 \mathrm{GHz}$ optical input pulses into $2.5 \mathrm{GHz}$ pulses using the PALM.

The experimental set-up for the all optical demultiplexing using a PALM is shown in Fig. 1. A PALM was constructed with a $10 \mathrm{~km}$-long dispersion shifted fiber with a zero-dispersion wavelength of $1545 \mathrm{~nm}$ used as the nonlinear medium, a 15-m long dispersion compensa-

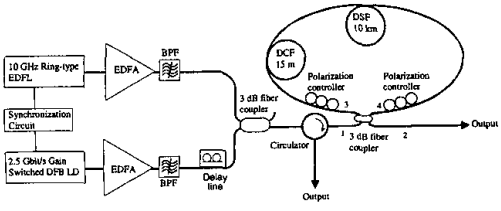

CWF75 Fig. 1. Experimental set-up.

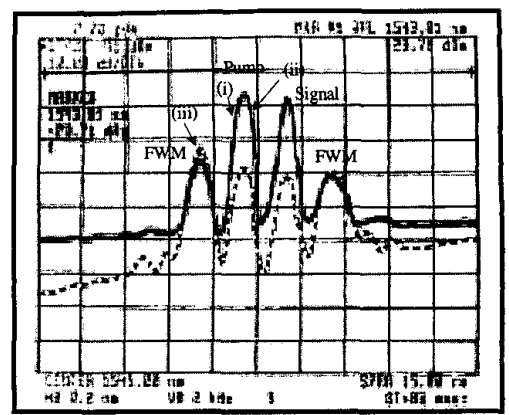

CWF75 Fig. 2. Optical spectra from the port 3 and 4 in PALM (curves (i) and (ii)) and port 2 of PALM (curve (iii)).

tion fiber with a group velocity dispersion of $-72.9 \mathrm{ps} /(\mathrm{nm} . \mathrm{km})$ at $1550 \mathrm{~nm}$ used as the dispersion element, two polarization controllers and a $3 \mathrm{~dB}$ fiber coupler. 10-ps signal pulses of $1546.0 \mathrm{~nm}$ wavelength were generated from a $10 \mathrm{GHz}$ wavelength tunable $\mathrm{Er}^{3+}$. doped fiber ring laser. 30 ps control pulses of $1544.5 \mathrm{~nm}$ were generated from a $2.5 \mathrm{GHz}$ gain switched DFB laser diode. Both signal and control pulses were amplified by EDFAs. The variable delay line located in the control signal input port was used to synchronize the control pulses with the signal pulses in the time domain. A fiber circulator was used to extract out and to monitor the reflected signal from the PALM. The input control and signal powers launched into the PALM were $11 \mathrm{~mW}$ and $10 \mathrm{~mW}$, respectively.

Figure 2 shows the observed output spectra of the PALM. The curves (i) and (ii) represent the observed spectra at ports 3 and 4 of the PALM, respectively. In these cases, the optical powers of the control and the signal waves are noticeably larger than that of the generated FWM waves. The observed spectrum measured at the port 2 of the PALM shown in curve (iii) of the Fig. 2 displays two FWM generated peaks at $1543.0 \mathrm{~nm}$ and $1547.5 \mathrm{~nm}$.

The residual components of the signal and control waves were suppressed by $>23 \mathrm{~dB}$ without an optical filtering, while the generated FWM wave's power increased by $3 \mathrm{~dB}$ after passing through the PALM. Therefore, the FWM wave can be separated from the control and signal waves without a bandpass filter. The oscilloscope sampling traces shown in Figs. 3(a) and 3(b) correspond to the curves (i) and (iii) of Fig. 2, respectively. The sampling trace measured with an open-loop at the dispersion shifted fiber position shows simultaneous presence of the generated FWM wave and the $10 \mathrm{GHz}$ signal and the $2.5 \mathrm{GHz}$ control waves. In this case, the optical bandpass filter is needed to separate the demultiplexed signal 